\title{
Spin diffusion and torques in disordered antiferromagnets
}

\author{
Aurelien Manchon ${ }^{1}$ \\ Physical Science and Engineering Division (PSE), King Abdullah University of Science and Technology \\ (KAUST), Thuwal 23955-6900, Saudi Arabia \\ E-mail: aurelien.manchon@kaust.edu.sa \\ Received 30 July 2016, revised 3 November 2016 \\ Accepted for publication 6 December 2016 \\ Published 31 January 2017

\begin{abstract}
We have developed a drift-diffusion equation of spin transport in collinear bipartite metallic antiferromagnets. Starting from a model tight-binding Hamiltonian, we obtain the quantum kinetic equation within Keldysh formalism and expand it to the lowest order in spatial gradient using Wigner expansion method. In the diffusive limit, these equations track the spatio-temporal evolution of the spin accumulations and spin currents on each sublattice of the antiferromagnet. We use these equations to address the nature of the spin transfer torque in (i) a spin-valve composed of a ferromagnet and an antiferromagnet, (ii) a metallic bilayer consisting of an antiferromagnet adjacent to a heavy metal possessing spin Hall effect, and in (iii) a single antiferromagnet possessing spin Hall effect. We show that the latter can experience a self-torque thanks to the non-vanishing spin Hall effect in the antiferromagnet.
\end{abstract} \\ Abstract
}

Keywords: spintronics, spin transfer torque, antiferromagnets

(Some figures may appear in colour only in the online journal)

Antiferromagnets $[1,2]$ (AF) have long remained an intriguing and exotic state of matter, whose application has been restricted to enabling interfacial exchange bias [3] in metallic and tunneling spin-valves [4]. Their role in the expanding field of applied spintronics has been mostly passive, and the in-depth investigation of their basic properties considered as fundamental condensed matter physics (see, e.g. [5-7]). A conceptual breakthrough was achieved ten years ago with the proposal that spin transfer torque could be used to electrically control the direction of the order parameter of antiferromagnetic spin valves, henceforth making these materials potential candidates for low energy spin devices [8]. In spite of substantial theoretical efforts and experimental attempts to observe such a torque, the difficulty of independently detecting the direction of the antiferromagnetic order parameter has remained a major obstacle. The paradigm has changed radically in the past few years with the discovery of antiferromagnetic anisotropic

\footnotetext{
${ }^{1}$ This article belongs to the special issue: emerging leaders, which features invited work from the best early-career researchers working within the scope of Journal of Physics: Condensed Matter. This project is part of the Journal of Physics Series' 50th anniversary celebrations in 2017. Aurelien Manchon was selected by the Editorial Board of Journal of Physics: Condensed Matter as an Emerging Leader.
}

(tunneling) magnetoresistance [9, 10], demonstrating that spin-orbit coupled antiferromagnets might emerge as the next frontier in applied spintronics, combining the promises of spinorbitronics [11] and the richness of antiferromagnets [12, 13].

Uncovering the nature of spin torque in antiferromagnetic devices has attracted numerous experimental and theoretical studies. From an experimental standpoint, in spite of an early observation of current-driven change in the exchange bias of a conventional metallic spin-valve [14, 15], a major breakthrough was achieved last year with the observation of a current-driven order parameter switching mediated by spin-orbit coupling in CuMnAs [16, 17]. Indeed, although CuMnAs is a collinear antiferromagnet with a centrosymmetric crystal structure, it possesses local inversion symmetry breaking: the two magnetic sublattices are space-inversion partners, enabling spin-orbit torques [16]. Encouraging results have also been obtained by Reichlová et al [18] in Ta/IrMn/CoFeB multilayer stack. From a theoretical perspective, besides a recent derivation of the spin-orbit torque [17], the physics of spin torque in antiferromagnetic spin devices has been mostly addressed numerically either through tight-binding [8, 19-26] or ab initio methods $[27,28]$. These works demonstrate, without ambiguity, that quantum coherence is a crucial ingredient for a sizable spin 
(a)

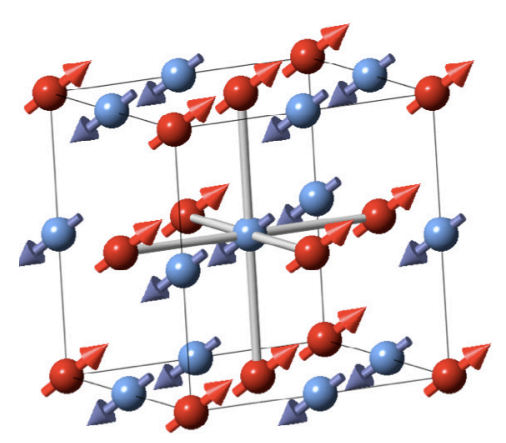

(b)

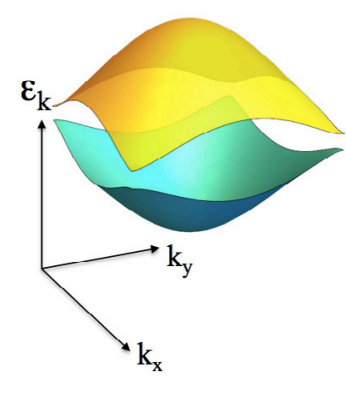

Figure 1. (a) Crystal structure of a prototypical G-type antiferromagnet. The red and blue sites and arrows denote the $A$ and $B$ sites and their magnetic moments. The light grey sticks represent the nearest neighbor hopping. (b) Band structure of the G-type antiferromagnet for $k_{z}=0$.

transfer torque in antiferromagnetic spin-valves [20, 22, 23]. The recent realization that the boundary condition at the interface between a normal metal and an antiferromagnet can be modeled through spin mixing conductance $[29,30]$ enables the phenomenological treatment of interfacial phenomena such as spin torque and spin pumping [31, 32].

Nevertheless, the spin transport in antiferromagnetic devices is still lacking a semiclassical theory that encompasses the important physical parameters governing spin drift and diffusion inside such devices. A drift-diffusion theory would prove very useful to model the spin transport in metallic systems involving antiferromagnets, and thereby complete the drift-diffusion theory already available for ferromagnets [33-35]. In this work, starting from a tight-binding Hamiltonian we derive such drift-diffusion equations for a G-type (checkerboard) antiferromagnet and apply the obtained equations to compute the spin transfer torque in three selected cases of interest for experiments: (i) a spin-valve composed of a ferromagnet and an antiferromagnet, (ii) a metallic bilayer consisting of an antiferromagnet adjacent to a heavy metal possessing spin Hall effect, and (iii) a single antiferromagnet possessing spin Hall effect.

\section{Antiferromagnetic dynamics and torque definition}

Before deriving the drift-diffusion equation in an antiferromagnet, let us first comment on the nature of the spin transfer torque in these materials. For simplicity, we look at a bipartite antiferromagnet composed of two sublattices $A$ and $B$, as illustrated in figure 1(a). The classical dynamics of such an antiferromagnet under a flowing current has already been investigated by Gomonay and Loktev [36, 37], but it is instructive to remember some of the most important aspects of it. Let us consider that each sublattice $i$ is described by LandauLifshitz-Gilbert (LLG) equation

$$
\begin{aligned}
\partial_{t} \mathbf{m}_{i}= & -\gamma \mathbf{m}_{i} \times \mathbf{H}-\gamma H_{\mathrm{K}}\left(\mathbf{m}_{i} \cdot \mathbf{u}\right) \mathbf{m}_{i} \times \mathbf{u} \\
& +\gamma H_{E} \mathbf{m}_{i} \times \mathbf{m}_{\bar{i}}+\alpha \mathbf{m}_{i} \times \partial_{t} \mathbf{m}_{i}+\mathbf{T}_{i} .
\end{aligned}
$$

Here $\mathbf{m}_{i}$ is the unit vector of the magnetization direction of sublattice $i, \gamma$ is the absolute value of the gyromagnetic ratio, $H_{E}$ is the antiferromagnetic exchange field (about $100 \mathrm{~T}$ in conventional antiferromagnets), $H_{\mathrm{K}}$ is the magnetic anisotropy field along direction $\mathbf{u}, \bar{i}$ is the complementary sublattice and $\mathbf{T}_{i}$ is the torque exerted on the lattice $i$ by a flowing charge current. In general, it is always possible to express $\mathbf{T}_{i}$ as

$$
\mathbf{T}_{i}=T_{\|}^{i} \mathbf{m}_{i} \times\left(\mathbf{p} \times \mathbf{m}_{i}\right)+T_{\perp}^{i} \mathbf{m}_{i} \times \mathbf{p},
$$

where $\mathbf{p}$ is a unit vector defined by the spin-polarization mechanism (external polarizer, spin Hall effect, Rashba effect etc). In the following we choose $\partial_{t} \mathbf{p}=0$ for simplicity. The first term $T_{\|}^{i}$ is sometimes called the in-plane torque as it lies in the $\left(\mathbf{p}, \mathbf{m}_{i}\right)$ plane, or the damping-like torque as it acts like a magnetic damping on the magnetic moment $\mathbf{m}_{i}$ in equation (1). The second term $T_{\perp}^{i}$ is sometimes called the out-of-plane torque as it lies perpendicular to the $\left(\mathbf{p}, \mathbf{m}_{i}\right)$ plane, or the field-like torque as it acts like a magnetic field on the magnetic moment $\mathbf{m}_{i}$ in equation (1). In the following discussion, we will show that the overall action of the torque components $T_{\|, \perp}^{i}$ on the Néel order parameter does not only depend on their direction (i.e. either $\sim \mathbf{m}_{i} \times\left(\mathbf{p} \times \mathbf{m}_{i}\right)$ or $\left.\sim \mathbf{m}_{i} \times \mathbf{p}\right)$, but also on their sublattices' symmetry (either uniform $\mathbf{T}_{A}=\mathbf{T}_{B}$ or staggered $\mathbf{T}_{A}=-\mathbf{T}_{B}$ ). Therefore, in the following, we denote $T_{\|}^{i}$ and $T_{\perp}^{i}$ as in-plane and out-of-plane torques, respectively, rather than damping-like and field-like torques.

The antiferromagnet can be characterized by the Néel order parameter, $\mathbf{n}=\left(\mathbf{m}_{A}-\mathbf{m}_{B}\right) / 2$, and the magnetization $\mathbf{m}=\left(\mathbf{m}_{A}+\mathbf{m}_{B}\right) / 2$. Combining the LLG equations for $A$ and $B$ sublattices yields a coupled equation for both $\mathbf{n}$ and $\mathbf{m}$. Since the procedure is quite standard and cumbersome, we only provide the final result for the dynamics of $\mathbf{n}$. This is done by assuming that $\mathbf{m}$ is a slave variable, an assumption valid for large exchange fields. More precisely, one can show that

$$
\begin{aligned}
2 \gamma H_{E} \mathbf{m}= & \partial_{t} \mathbf{n} \times \mathbf{n}+\gamma \mathbf{n} \times(\mathbf{H} \times \mathbf{n})-\frac{1}{2}\left(T_{\perp}^{A}+T_{\perp}^{B}\right) \mathbf{n} \\
& \times(\mathbf{p} \times \mathbf{n})+\frac{1}{2}\left(T_{\|}^{A}-T_{\|}^{B}\right) \mathbf{n} \times \mathbf{p} .
\end{aligned}
$$

This equation states that an external magnetic field $\mathbf{H}$ can induce a magnetization $\mathbf{m}$ (second term) that remains very small as long as $|\mathbf{H}| \ll H_{E}$. In addition, in-plane and outof-plane staggered torques, such as $\mathbf{T}_{A}=-\mathbf{T}_{B}$, can also induce magnetization. To the first order in magnetization $\mathbf{m}$, the dynamics equation of the Néel order parameter reads

$$
\begin{aligned}
\partial_{t}^{2} \mathbf{n} \times \mathbf{n}= & -2 \gamma^{2} H_{\mathrm{E}} H_{\mathrm{K}}(\mathbf{n} \cdot \mathbf{u}) \mathbf{n} \times \mathbf{u}+\gamma^{2}(\mathbf{n} \cdot \mathbf{H}) \mathbf{n} \\
& \times \mathbf{H}+2 \gamma H_{\mathrm{E}} \alpha \mathbf{n} \times \partial_{t} \mathbf{n}+2 \gamma(\mathbf{n} \cdot \mathbf{H}) \partial_{t} \mathbf{n} \\
& +\gamma H_{\mathrm{E}}\left(T_{\|}^{A}+T_{\|}^{B}\right) \mathbf{n} \times(\mathbf{p} \times \mathbf{n})+\gamma H_{\mathrm{E}}\left(T_{\perp}^{A}-T_{\perp}^{B}\right) \mathbf{n} \times \mathbf{p} \\
& -\frac{1}{2} \partial_{t}\left(T_{\|}^{A}-T_{\|}^{B}\right) \mathbf{n} \times \mathbf{p}+\frac{1}{2} \partial_{t}\left(T_{\perp}^{A}+T_{\perp}^{B}\right) \mathbf{n} \times(\mathbf{p} \times \mathbf{n}) \\
& -\gamma \mathbf{n} \times\left(\partial_{t} \mathbf{H} \times \mathbf{n}\right)-\frac{1}{2}\left(T_{\|}^{A}-T_{\|}^{B}\right) \partial_{t} \mathbf{n} \times \mathbf{p} \\
& -\frac{1}{2}\left(T_{\perp}^{A}+T_{\perp}^{B}\right) \partial_{t}[(\mathbf{n} \cdot \mathbf{p}) \mathbf{n}]
\end{aligned}
$$


The first line describes the usual dynamics of antiferromagnets [38]. The magnetic field acts on the Néel order parameter at the second order (second term) and through an additional friction term, $\sim \partial_{t} \mathbf{n}$ (fourth term). Most importantly, the fifth and sixth terms show that the in-plane and out-of-plane components of the torque induce Néel order dynamics only as long as their magnitude is uniform on the sublattices [36], i.e. $\mathbf{T}_{A}=\mathbf{T}_{B}$. This means that the ability of the spin transfer torque to excite the Néel order parameter does not depend on whether the torque lies in or out of the $(\mathbf{n}, \mathbf{p})$ plane, but rather on whether the torque magnitude itself is staggered or uniform on opposite sublattices. This is an essential feature of currentdriven antiferromagnetic dynamics already noticed in $[12,17$, 37]. The seventh, eighth and ninth terms states that a timedependent uniform magnetic field (or staggered spin torque) can affect the dynamics of the Néel order parameter. Such a dynamic has been illustrated by the recent demonstration of Néel order manipulation using ultrashort laser pulses $[39,40]$. Finally, the last two terms show that staggered torques contribute to both the kinetic energy and energy dissipation.

To summarize this section, one should always keep in mind that in antiferromagnets the torques that are efficient in manipulating the Néel order parameter must have the same sign on opposite sublattices, $\mathbf{T}_{A}=\mathbf{T}_{B}$ (uniform torques). In contrast, torques that change sign on opposite sublattices, $\mathbf{T}_{A}=-\mathbf{T}_{B}$, (staggered torques) can manipulate the Néel order through their time derivative, i.e. using electrical pulses for instance. Notice that it is always possible to express a torque $\mathbf{T}_{i}$ in terms of a local effective field $\mathbf{h}_{i}$, such that $\mathbf{T}_{i}=-\gamma \mathbf{m}_{i} \times \mathbf{h}_{i}$. Hence, following [12, 17], uniform torques are associated with staggered effective fields, while staggered torques are associated with uniform effective fields. So, equivalently, the Néel order parameter can be manipulated either by time-independent staggered effective fields or by time-dependent uniform effective fields.

\section{Drift-diffusion equations in antiferromagnets}

\subsection{Four band model}

Let us now turn our attention towards spin transport in disordered antiferromagnets. We consider a $\mathrm{NaCl}$ crystal lattice composed of two interpenetrating fcc sublattices, $A$ and $B$, whose magnetic moments are antiferromagnetically coupled and aligned along the direction of the order parameter $\mathbf{n}$, as illustrated in figure 1(a). In this work, we consider only nearest neighbor hopping and neglect next-nearest neighbor hopping. Furthermore, in the following we consider that the dynamics of the Néel order parameter $\mathbf{n}$ is slow compared to the itinerant spin dynamics, such that $\partial_{t} \mathbf{n} \approx 0$. The extension of the present method to layered antiferromagnets is also possible, but remains out of the scope of the present study. The nearest-neighbor tight-binding Hamiltonian in the $(|A\rangle,|B\rangle) \otimes$ $(|\uparrow\rangle,|\downarrow\rangle)$ basis reads

$$
\tilde{H}_{0}=\gamma_{k} \hat{\tau}_{x} \otimes \hat{1}+\Delta \hat{\tau}_{z} \otimes \hat{\boldsymbol{\sigma}} \cdot \mathbf{n},
$$

$$
\gamma_{k}=-2 t_{\mathrm{N}} \sum_{i \neq j} \cos \frac{k_{i} a}{2} \cos \frac{k_{j} a}{2}
$$

where $\hat{\boldsymbol{\tau}}$ and $\hat{\boldsymbol{\sigma}}$ are the vectors of $2 \times 2$ spin Pauli matrices referring to the sublattice and spin angular momentum spaces, respectively, $t_{\mathrm{N}}$ is the nearest neighbor hopping integral and $\Delta$ is the exchange energy between the itinerant electrons and the localized electrons. Symbols $\wedge$ and $\sim$ denote $2 \times 2$ and $4 \times 4$ operators, respectively. Close to $\Gamma$-point, $\mathbf{k} \rightarrow 0$ and $\gamma_{k} \rightarrow t_{\mathrm{N}} a^{2} k^{2}-6 t_{\mathrm{N}}$. In the presence of spin-independent impurities, we obtain the following Hamiltonian

$$
\tilde{H}=\gamma_{k} \hat{\tau}_{x} \otimes \hat{1}+\Delta \hat{\tau}_{z} \otimes \hat{\boldsymbol{\sigma}} \cdot \mathbf{n}+\tilde{V}(\mathbf{r}) .
$$

The impurity potential consists of short range (delta-like) potentials randomly distributed over the volume of the crystal,

$\tilde{V}(\mathbf{r})=\frac{1}{2}\left(1+\hat{\tau}_{z}\right) \sum_{i \in \Omega_{A}} V_{0} \delta\left(\mathbf{r}-\mathbf{r}_{i}\right)+\frac{1}{2}\left(1-\hat{\tau}_{z}\right) \sum_{i \in \Omega_{B}} V_{0} \delta\left(\mathbf{r}-\mathbf{r}_{i}\right)$,

where the operators $\frac{1}{2}\left(1 \pm \hat{\tau}_{z}\right)$ refer to the $A$ and $B$ sublattice, respectively, and the summation runs over the volume occupied by each sublattice, $\Omega_{A, B}$. This form ensures that disorder on sublattice $A$ is not correlated with disorder on sublattice $B$.

The unperturbed retarded (advanced) Green's function associated with $\tilde{H}_{0}$ is defined as $\tilde{G}_{0}^{R, A}=\left(\epsilon-\tilde{H}_{0} \pm \mathrm{i} \eta\right)^{-1}$, and reads explicitly

$$
\tilde{G}_{k, 0}^{R, A}=\frac{1}{2} \sum_{\nu= \pm 1} \frac{\tilde{1}+\nu \cos \chi_{k} \hat{\tau}_{x} \otimes \hat{1}+\nu \sin \chi_{k} \hat{\tau}_{z} \otimes \hat{\boldsymbol{\sigma}} \cdot \mathbf{n}}{\epsilon-\epsilon_{k, \nu} \pm \mathrm{i} 0^{+}}
$$

where $\nu= \pm 1$ is the band index, and

$$
\epsilon_{k, \nu}=\nu \sqrt{\gamma_{k}^{2}+\Delta^{2}}, \cos \chi_{k}=\frac{\gamma_{k}}{\sqrt{\gamma_{k}^{2}+\Delta^{2}}}, \sin \chi_{k}=\frac{\Delta}{\sqrt{\gamma_{k}^{2}+\Delta^{2}}} .
$$

With the notation given above, the gap is centered at $\epsilon=0$. From now on, we consider the bottom band $(\nu=-1)$ and the electron energy will be negative, $-\sqrt{\left(6 t_{\mathrm{N}}\right)^{2}+\Delta^{2}}<\epsilon<-\Delta$. As a result, in the reciprocal space and within first Born approximation, the lesser, retarded and advanced self-energies read

$$
\tilde{\Sigma}^{<, R, A}=\int \frac{\mathrm{d}^{3} \mathbf{k}}{(2 \pi)^{3}} \tilde{V}_{k} \tilde{G}_{k}^{<, R, A} \tilde{V}_{k},
$$

$\tilde{V}_{k}$ being the Fourier transform of $\tilde{V}(\mathbf{r})$. More explicitly

$$
\begin{gathered}
\tilde{\Sigma}^{R, A}=\mp \frac{\mathrm{i}}{4 \tau}\left(\tilde{1}-\beta \hat{\tau}_{z} \otimes \hat{\boldsymbol{\sigma}} \cdot \mathbf{n}\right), \\
\tilde{\Sigma}^{<}=\frac{1}{4 \pi \mathcal{N} \tau}\left(\left\langle\tilde{G}_{k}^{<}\right\rangle+\hat{\tau}_{z}\left\langle\tilde{G}_{k}^{<}\right\rangle \hat{\tau}_{z}\right) .
\end{gathered}
$$

Here, we defined the density of states $\mathcal{N}=\int \mathrm{d}^{3} \mathbf{k} /(2 \pi)^{3} \delta\left(\epsilon-\epsilon_{k}\right)$, and the momentum relaxation time $1 / \tau=2 \pi n_{i} \mathcal{N} \mid V_{0}^{2}$, where $n_{i}$ is the impurity concentration, and $\beta=\cos \chi_{k_{\mathrm{F}}}$ is the onsite polarization at Fermi energy. Finally, $\left\langle\tilde{G}_{k}^{<}\right\rangle=\int \mathrm{d}^{3} \mathbf{k} /(2 \pi)^{3} \tilde{G}_{k}^{<}$. 


\subsection{Quantum kinetics}

Let us now derive the equation of motion of electrons flowing in the antiferromagnet. To do so, we start from Dyson equation,

$$
\left[\hat{\mathcal{G}}^{R}\right]^{-1} \star \hat{\mathcal{G}}^{<}-\hat{\mathcal{G}}^{<} \star\left[\hat{\mathcal{G}}^{A}\right]^{-1}=\hat{\mathcal{S}}^{<} \star \hat{\mathcal{G}}^{A}-\hat{\mathcal{G}}^{R} \star \hat{\mathcal{S}}^{<}
$$

where the Green's functions $\tilde{\mathcal{G}}=\tilde{\mathcal{G}}\left(\mathbf{r}, \mathbf{r}^{\prime} ; t, t^{\prime}\right)$ and self-energies $\tilde{\mathcal{S}}=\tilde{\mathcal{S}}\left(\mathbf{r}, \mathbf{r}^{\prime} ; t, t^{\prime}\right)$ are defined in real space and time, and $\star$ is the convolution product in both space and time. We then perform a Wigner expansion in the center of mass coordinates $\left(\left(\mathbf{r}+\mathbf{r}^{\prime}\right) / 2,\left(t+t^{\prime}\right) / 2\right)$, while the short range variations $\left(\mathbf{r}-\mathbf{r}, t-t^{\prime}\right)$ are Fourier transformed. This method allows for the rewriting of the Dyson equation in k-space to the lowest order in spatial and temporal gradients, thereby separating the (real space-time) semiclassical dynamics from the (reciprocal space-time) quantum effects. Since this method has been described in detail in several publications [41-46], we directly provide the kinetic equation

$$
\mathrm{i} \hbar \partial_{t} \tilde{G}_{k}^{<}+\left[\tilde{G}_{k}^{<}, \tilde{H}_{0}\right]+\mathrm{i}\left\{\tilde{G}_{k}^{<}, \tilde{\Sigma}\right\}+\frac{\mathrm{i}}{2}\left\{\hat{v}_{i}, \partial_{i} \tilde{G}_{k}^{<}\right\}=\tilde{\Sigma}^{<} \tilde{G}_{k}^{A}-\tilde{G}_{k}^{R} \tilde{\Sigma}^{<},
$$

where we defined $\hat{\Sigma}^{R, A}=\mp \mathrm{i} \hat{\Sigma}$, and the velocity operator $\hat{v}_{i}=\partial_{\hbar k_{i}} \tilde{H}_{0}$. In order to obtain sublattice-resolved coupled equations, we now expand the lesser Green's function $\tilde{G}_{k}^{<}$in the sublattice basis. We write

$$
\tilde{G}_{k}^{<}=\frac{1}{2}\left(1+\hat{\tau}_{z}\right) \otimes \hat{g}_{k}^{A}+\frac{1}{2}\left(1-\hat{\tau}_{z}\right) \otimes \hat{g}_{k}^{B}+\hat{\tau}_{x} \otimes \hat{g}_{k}^{x}+\hat{\tau}_{y} \otimes \hat{g}_{k}^{y} .
$$

Here, $\hat{g}_{k}^{\alpha}$ is a $2 \times 2$ spinor in the spin basis, $\hat{g}_{k}^{A, B}$ are the components of the lesser Green's function on sublattice $A$ and $B$, while $\hat{g}_{k}^{x, y}$ contain the off-diagonal elements that connect the two sublattices. In [21], this connection is accounted for through a Berry curvature in the subband space. Our procedure is different as we explicitly expand the kinetic equations in the sublattice space, but the physics captured is essentially the same. Hence, by taking the trace of equation (15) over the components of $\hat{\boldsymbol{\tau}}$, one obtains four coupled equations

$$
\begin{gathered}
\partial_{t} \hat{g}_{k}^{A}-\frac{2 \gamma_{k}}{\hbar} \hat{g}_{k}^{y}-\mathrm{i} \frac{\Delta}{\hbar}\left[\hat{g}_{k}^{A}, \hat{\boldsymbol{\sigma}} \cdot \mathbf{n}\right]+v_{i} \partial_{i} \hat{g}_{k}^{x}=\frac{1}{2 \tau}\left(\left\langle\hat{g}_{k}^{A}\right\rangle \delta_{k}-\hat{g}_{k}^{A}\right) \\
-\frac{\beta}{4 \tau}\left\{\left\langle\hat{g}_{k}^{A}\right\rangle \delta_{k}-\hat{g}_{k}^{A}, \hat{\boldsymbol{\sigma}} \cdot \mathbf{n}\right\}, \\
\partial_{t} \hat{g}_{k}^{B}+\frac{2 \gamma_{k}}{\hbar} \hat{g}_{k}^{y}+\mathrm{i} \frac{\Delta}{\hbar}\left[\hat{g}_{k}^{B}, \hat{\boldsymbol{\sigma}} \cdot \mathbf{n}\right]+v_{i} \partial_{i} \hat{g}_{k}^{x}=\frac{1}{2 \tau}\left(\left\langle\hat{g}_{k}^{B}\right\rangle \delta_{k}-\hat{g}_{k}^{B}\right) \\
+\frac{\beta}{4 \tau}\left\{\left\langle\hat{g}_{k}^{B}\right\rangle \delta_{k}-\hat{g}_{k}^{B}, \hat{\boldsymbol{\sigma}} \cdot \mathbf{n}\right\}, \\
\partial_{t} \hat{g}_{k}^{x}+\frac{1}{2 \tau} \hat{g}_{k}^{x}+\frac{\Delta}{\hbar}\left\{\hat{g}_{k}^{y}, \hat{\boldsymbol{\sigma}} \cdot \mathbf{n}\right\}-\frac{\mathrm{i} \beta}{4 \tau}\left[\hat{g}_{k}^{y}, \hat{\boldsymbol{\sigma}} \cdot \mathbf{n}\right] \\
\quad+\frac{v_{i}}{2} \partial_{i}\left(\hat{g}_{k}^{A}+\hat{g}_{k}^{B}\right)=0,
\end{gathered}
$$

$$
\partial_{t} \hat{g}_{k}^{y}+\frac{1}{2 \tau} \hat{g}_{k}^{y}+\frac{\gamma_{k}}{\hbar}\left(\hat{g}_{k}^{A}-\hat{g}_{k}^{B}\right)-\frac{\Delta}{\hbar}\left\{\hat{g}_{k}^{x}, \hat{\boldsymbol{\sigma}} \cdot \mathbf{n}\right\}+\frac{\mathrm{i} \beta}{4 \tau}\left[\hat{g}_{k}^{x}, \hat{\boldsymbol{\sigma}} \cdot \mathbf{n}\right]=0 .
$$

We define $\delta_{k}=\delta\left(\epsilon-\epsilon_{k}\right) / \mathcal{N}$. The electric field $\mathbf{E}$ can be installed by performing the substitution $v_{i} \partial_{i} \rightarrow v_{i} \partial_{i}-e E_{i} v_{i} \partial_{\epsilon}$. Equations (17)-(20) constitute the semiclassical basis on which the drift-diffusion theory is built. At this stage, one could solve these equations numerically and obtain the momentumresolved non-equilibrium distributions $\hat{g}_{k}^{A, B, x}$ [47]. Such a procedure allows for the proper modeling of systems with a size of the order of the mean free path $\lambda \sim v_{\mathrm{F}} \tau$ ( $v_{\mathrm{F}}$ being the Fermi velocity), where semiclassical size effects take place. In the present work, we are interested in systems that are much larger than the mean free path and for which we can obtain explicit transport equations.

\subsection{Drift-diffusion equations}

In order to obtain analytically solvable equations, we need to get rid of the momentum dependence and perform a momentum averaging. By doing so, we assume that any spatial dependence (size of the system and density gradients) is much larger than the mean free path $\lambda$. Hence, any effect that takes place on a scale of the order of the mean free path is disregarded [33]. This procedure reduces the semiclassical equations (17)-(20) to a set of explicit drift-diffusion equations.

We define the density matrices on sublattices $A$ and $B$ as $\hat{\rho}_{A, B}=\int \mathrm{d}^{3} \mathbf{k} /(2 \pi)^{3} \hat{g}_{k}^{A, B} / 2 \pi \mathcal{N}$ and the current density spinor as $\hat{\mathcal{J}}_{i}=\int \mathrm{d}^{3} \mathbf{k} /(2 \pi)^{3} v_{i} \hat{g}_{k}^{x} / \pi \mathcal{N}$. We also define the auxiliary quantities $\hat{\rho}_{y}=\int \mathrm{d}^{3} \mathbf{k} /(2 \pi)^{3} \hat{g}_{k}^{y} / 2 \pi \mathcal{N}$ and $\hat{\mathcal{J}}_{i}^{y}=\int \mathrm{d}^{3} \mathbf{k} /(2 \pi)^{3} v_{i} \hat{g}_{k}^{y} / 2 \pi \mathcal{N}$. These last two quantities do not have a straightforward physical meaning and can be eliminated to derive diffusion equations involving only $\hat{\rho}_{A, B}$ and $\hat{\mathcal{J}}_{i}$. After some algebra, one obtains

$$
\left(1+\frac{\xi^{2}}{2}-\frac{\beta^{2}}{2}\right) \hat{\mathcal{J}}_{i}+\left(\frac{\xi^{2}}{2}+\frac{\beta^{2}}{2}\right) \hat{\boldsymbol{\sigma}} \cdot \mathbf{n} \hat{\mathcal{J}}_{i} \hat{\boldsymbol{\sigma}} \cdot \mathbf{n}=-\mathcal{D} \partial_{i}\left(\hat{\rho}_{A}+\hat{\rho}_{B}\right)
$$

$$
\begin{aligned}
& \partial_{t} \hat{\rho}_{A}+\frac{\Gamma}{\hbar}\left(\hat{\rho}_{A}-\hat{\rho}_{B}\right)-\mathrm{i} \frac{\Delta}{\hbar}\left[\hat{\rho}_{A}, \hat{\boldsymbol{\sigma}} \cdot \mathbf{n}\right]=-\frac{1}{2} \partial_{i} \hat{\mathcal{J}}_{i}, \\
& \partial_{t} \hat{\rho}_{B}-\frac{\Gamma}{\hbar}\left(\hat{\rho}_{A}-\hat{\rho}_{B}\right)+\mathrm{i} \frac{\Delta}{\hbar}\left[\hat{\rho}_{B}, \hat{\boldsymbol{\sigma}} \cdot \mathbf{n}\right]=-\frac{1}{2} \partial_{i} \hat{\mathcal{J}}_{i} .
\end{aligned}
$$

Here $\mathcal{D}=2 \tau v_{\mathrm{F}}^{2} / 3$ is the diffusion coefficient, $\xi=4 \tau \Delta / \hbar$, and $\Gamma=4 \tau \epsilon^{2}\left(1-\beta^{2}\right)$. Finally, by recognizing that $\hat{\mathcal{J}}_{i}=$ $j_{c, i}+\hat{\boldsymbol{\sigma}} \cdot \mathbf{J}_{i}^{s}$, where $j_{c, i}$ is the charge current density and $\mathbf{J}_{i}^{s}$ the spin current density flowing along the direction of the unit vector $\mathbf{e}_{i}$, and $\hat{\rho}_{\alpha}=n_{\alpha}+\hat{\boldsymbol{\sigma}} \cdot \mathbf{S}_{\alpha}$, where $n_{\alpha}$ and $\mathbf{S}_{\alpha}$ are the charge and spin densities on sublattice $\alpha$, we obtain

$$
\partial_{t} n_{A}+\frac{\Gamma}{\hbar}\left(n_{A}-n_{B}\right)=-\frac{1}{2} \partial_{i} j_{c, i}
$$




$$
\begin{gathered}
\partial_{t} n_{B}-\frac{\Gamma}{\hbar}\left(n_{A}-n_{B}\right)=-\frac{1}{2} \partial_{i} j_{c, i}, \\
\partial_{t} \mathbf{S}_{A}+\frac{\Gamma}{\hbar}\left(\mathbf{S}_{A}-\mathbf{S}_{B}\right)+\frac{2 \Delta}{\hbar} \mathbf{S}_{A} \times \mathbf{n}+\frac{1}{\tau_{\mathrm{sf}}} \mathbf{S}_{A}=-\frac{1}{2} \partial_{i} \mathbf{J}_{i}^{s}, \\
\partial_{t} \mathbf{S}_{B}-\frac{\Gamma}{\hbar}\left(\mathbf{S}_{A}-\mathbf{S}_{B}\right)-\frac{2 \Delta}{\hbar} \mathbf{S}_{B} \times \mathbf{n}+\frac{1}{\tau_{\mathrm{sf}}} \mathbf{S}_{B}=-\frac{1}{2} \partial_{i} \mathbf{J}_{i}^{s} .
\end{gathered}
$$

Equations (24)-(27) describe the diffusion of the charge and spin densities on sublattices $A$ and $B$, in which spin relaxation $\sim 1 / \tau_{\mathrm{sf}}$ has been introduced by hand. The source of the charge/spin dynamics is given by the gradient of the charge and spin current, $\partial_{i} j_{c, i}$ and $\partial_{i} \mathbf{J}_{i}^{\boldsymbol{S}}$. The charge and spin currents are defined

$$
j_{c, i}=-\mathcal{D}^{\|} \partial_{i}\left(n_{A}+n_{B}\right),
$$

$$
\mathbf{J}_{i}^{s}=-\mathcal{D}^{\|} \partial_{i}\left[\left(\mathbf{S}_{A}+\mathbf{S}_{B}\right) \cdot \mathbf{n}\right] \mathbf{n}-\mathcal{D}^{\perp} \mathbf{n} \times\left[\partial_{i}\left(\mathbf{S}_{A}+\mathbf{S}_{B}\right) \times \mathbf{n}\right],
$$

In other words, the charge (spin) current $j_{c, i}\left(\mathbf{J}_{i}^{S}\right)$ is the total charge (spin) current flowing through the diatomic unit cell along the direction $\mathbf{e}_{i}$. Here, $\mathcal{D}^{\|}=\mathcal{D} /\left(1+\xi^{2}\right)$, $\mathcal{D}^{\perp}=\mathcal{D} /\left(1-\beta^{2}\right)$ (Notice that by definition, $|\epsilon|>\Delta$ ). Most importantly, the charge/spin dynamics on the two sublattices are coupled through a term $\sim \Gamma$. This term is proportional to the ratio between the energy broadening due to disorder $(\sim \hbar / \tau)$ and the kinetic energy of the carriers $\left(\left\langle\gamma_{k}\right\rangle\right)$ and as such, the coupling term $\Gamma$ accounts for the time a spin carrier spends on one sublattice. One can then foresee that there is an interesting interplay between the lifetime of the carrier on a certain sublattice, $\hbar / \Gamma$, and the spin precession time, $\tau_{\Delta}=\hbar / 2 \Delta$.

This interplay arises explicitly by manipulating equations (26) and (27) in a more convenient form. In the limit of slow spin dynamics $(\hbar / \Gamma) \partial_{t} \ll 1$, we obtain

$$
\partial_{t}\left(n_{A}+n_{B}\right)=-\partial_{j} j_{c, i},
$$

$\partial_{t}\left(\mathbf{S}_{A}+\mathbf{S}_{B}\right)+\frac{1}{\tau_{\varphi}} \mathbf{n} \times\left[\left(\mathbf{S}_{A}+\mathbf{S}_{B}\right) \times \mathbf{n}\right]+\frac{1}{\tau_{\mathrm{sf}}}\left(\mathbf{S}_{A}+\mathbf{S}_{B}\right)=-\partial_{i} \mathbf{J}_{i}^{s}$,

$$
\mathbf{S}_{A}-\mathbf{S}_{B}=\frac{\tau^{*}}{\tau_{\Delta}} \mathbf{n} \times\left(\mathbf{S}_{A}+\mathbf{S}_{B}\right),
$$

where $1 / \tau^{*}=2 \Gamma / \hbar+1 / \tau_{\mathrm{sf}}$ and $\tau_{\varphi}=\tau_{\Delta}^{2} / \tau^{*}$. Equation (31) describes the diffusion of the uniform spin density $\mathbf{S}_{A}+\mathbf{S}_{B}$, while equation (32) defines the staggered spin density $\mathbf{S}_{A}-\mathbf{S}_{B}$. In equation (31), the third term on the left-hand side, $\sim 1 / \tau_{\mathrm{sf}}$, relaxes the (uniform) spin density isotropically, and the second term, $\sim 1 / \tau_{\varphi}$, relaxes only the component that is transverse to the magnetic order parameter $\mathbf{n}$. As such, this equation suggests that an antiferromagnet behaves like a normal metal with an anisotropic spin relaxation.

From the spin transfer torque standpoint, the staggered spin density $\mathbf{S}_{A}-\mathbf{S}_{B}$ is the most important quantity, as discussed in section 1 . The staggered spin density arises from the precession of the uniform spin density about the local order parameter $\mathbf{n}$. The magnitude of the staggered spin density is (a)

(c)

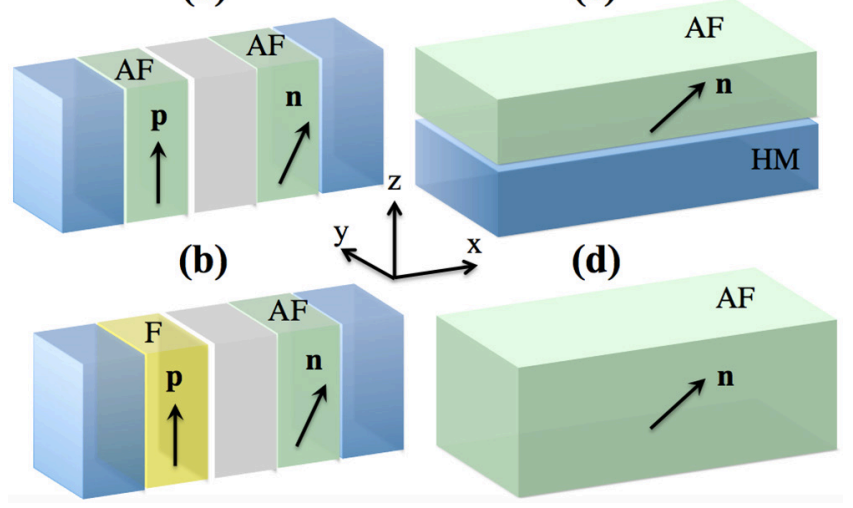

Figure 2. Schematics of four spin devices based on antiferromagnets: (a) a spin-valve composed of two antiferromagnets, (b) a spin-valve composed of a ferromagnetic polarizer and an antiferromagnet, (c) a bilayer composed of an antiferromagnet deposited on a heavy metal, and (d) a single antiferromagnet with spin-orbit coupling.

governed by the ratio between the carrier lifetime on one sublattice, $\sim \tau^{*}$, and the spin precession time, $\sim \tau_{\Delta}$. As a matter of fact, when the mobility is weak and carrier lifetime large compared to the spin precession time $\left(\tau^{*} \gg \tau_{\Delta}\right)$, the incoming spin has the time to precess about the local magnetic moment, thereby generating a staggered spin density. On the contrary, when the carrier lifetime is shorter than the spin precession time $\left(\tau^{*} \ll \tau_{\Delta}\right)$, the itinerant spin jumps quickly from one sublattice to the other without having the time to precess significantly. Therefore, the incoming spin density is weakly affected by the precession and the staggered spin density vanishes.

Finally, we emphasize that the drift-diffusion theory developed above neglects spin-orbit coupling effects both in bulk and at interfaces. Bulk effects can be added, for instance, in the form of Rashba spin-orbit coupling in equation (5), or spin-orbit coupled disorder in equation (8). The methods to treat spin-orbit coupling properly have been developed in the context of normal metals and ferromagnets [42-44] and its formal derivation in antiferromagnets is left to a future study. Spin-orbit coupling is also very important at interfaces as it relaxes the spin current flowing through. Such effects can be modeled by adding a fictitious interfacial layer with a phenomenological spin relaxation length (see e.g. [48]). In order to avoid overcomplicating our analytical results, we choose to disregard this effect in the remainder of the paper.

\section{Spin torque in devices based on antiferromagnets}

Let us now apply the drift-diffusion theory developed in the previous section to four devices involving antiferromagnets and illustrated in figure 2: (a) a spin-valve composed of two antiferromagnets, (b) a spin-valve composed of a ferromagnetic polarizer and an antiferromagnetic free layer, (c) a bilayer composed of an antiferromagnet deposited on top of a heavy metal and (d) an antiferromagnet with spin-orbit coupling. 


\subsection{Antiferromagnetic/antiferromagnetic spin-valve}

Such a spin-valve has been investigated numerically in several works $[8,20,23,28]$ and it is found that the currentdriven spin density is dominated by a uniform out-of-plane contribution, with a smaller staggered in-plane component. In other words, using our notation, $\mathbf{S}_{A}+\mathbf{S}_{B} \sim \mathbf{n} \times \mathbf{p}$, and $\mathbf{S}_{A}-\mathbf{S}_{B} \sim \mathbf{n} \times(\mathbf{p} \times \mathbf{n})$. However, these works point out that the non-equilibrium spin density only survives when quantum coherence is preserved [20, 23]. In these calculations, the non-equilibrium spin density arises from quantum-coherent reflections at the interfaces between the antiferromagnets. In the diffusive limit, equation (32), the staggered spin density stems as a correction to the uniform spin density $\mathbf{S}_{A}+\mathbf{S}_{B}$. In equation (31) the source of uniform spin density is given by the spin current gradient $-\partial_{i} \mathbf{J}_{i}^{S} \sim \partial_{i}^{2}\left(\mathbf{S}_{A}+\mathbf{S}_{B}\right)$. In other words, the uniform spin density can only be non-zero if there is a source of spin polarization of some sort (external spin polarizer, spin Hall effect etc). As a result, it is simply not possible to achieve spin transfer torque in a spin-valve composed of two antiferromagnets in the diffusive limit.

\subsection{Ferromagnetic/antiferromagnetic spin-valve}

Let us now consider a spin-valve composed of a ferromagnetic spin-polarizer and a 'free' antiferromagnet $[19,36]$, as depicted in figure 2(b). In this model, we consider two semi-infinite (ferro or antiferro)magnetic electrodes separated by a metallic spacer. The antiferromagnet has the usual G-type configuration discussed above, for which spin transport is governed by equations (31)-(32). To connect our drift-diffusion equations to that in ferromagnets $[34,35]$, we consider that the ferromagnet has a diatomic unit cell similar to the G-type antiferromagnet, with $A$ and $B$ sublattices. In contrast with the antiferromagnet though, in the ferromagnet the magnetic moments on $A$ and $B$ sublattices are ferromagnetically coupled. Hence, the usual spin diffusion equations apply on the total spin density $\mathbf{S}_{A}+\mathbf{S}_{B}$ of the ferromagnetic unit cell and read [34, 35]

$$
\begin{aligned}
& \mathbf{J}_{i}^{s}=-\mathcal{D P} \mathcal{N} e E_{i} \mathbf{m}-\mathcal{D} \partial_{i}\left(\mathbf{S}_{A}+\mathbf{S}_{B}\right), \\
& \partial_{t}\left(\mathbf{S}_{A}+\mathbf{S}_{B}\right)+\frac{1}{\tau_{\Delta}} \mathbf{m} \times\left(\mathbf{S}_{A}+\mathbf{S}_{B}\right)+\frac{1}{\tau_{\varphi}} \mathbf{m} \times\left[\left(\mathbf{S}_{A}+\mathbf{S}_{B}\right) \times \mathbf{m}\right] \\
& +\frac{1}{\tau_{\mathrm{sf}}}\left(\mathbf{S}_{A}+\mathbf{S}_{B}\right)=-\partial_{i} \mathbf{J}_{i}^{s} \text {. }
\end{aligned}
$$

Note that there is an important difference between the definition of spin current in a ferromagnet, equation (33), and its definition in an antiferromagnet, equation (29). In a ferromagnet, the density of states is spin-polarized (with a polarization factor $P$ ) and therefore a spin current can be generated simply by passing a charge current through it [33] (first term in equation (33)). In an antiferromagnet, the density of states is spin-polarized locally, but not globally (at the level of the unit cell) and hence, no spin current can be generated electrically. We also emphasize that equation (34) displays a precession term $\left(\sim 1 / \tau_{\Delta}\right)$ that is absent in antiferromagnets, equation (31).

We now simply need to connect the three layers. We make the following simplification: we assume that there is no interfacial spin relaxation between the layers (the spin current is continuous), and the spin relaxation in the spacer is much longer than the width of the spacer. We also consider that there is an interfacial resistance $r_{\text {int }}$ between the spacer and the antiferromagnet. We then obtain the uniform spin density in the ferromagnet,

$$
\begin{aligned}
\mathbf{S}_{A}+\left.\mathbf{S}_{B}\right|_{x<x_{L}}= & \frac{P j_{c}}{e^{3} \mathcal{N}} \frac{\mathrm{e}^{\left(x-x_{L}\right) / \lambda_{\|}^{L}}}{D}\left(\eta_{\|}\left[\eta_{\perp} \sigma_{\perp}^{L}+\sigma_{\perp}^{R}+\frac{\eta_{\perp}^{2} \sigma_{\Delta}^{L 2}}{\chi_{\perp} \sigma_{\perp}^{R}}\right]-\left(\eta_{\|} \sigma_{\perp}^{R}-\eta_{\perp} \sigma_{\|}^{R}\right) \sin ^{2} \theta\right) \mathbf{p} \\
& +\frac{P j_{c}}{e^{3} \mathcal{N}} \frac{\mathrm{e}^{\left(x-x_{L}\right) / \lambda_{\perp}^{L}}}{D}\left(\eta_{\|} \sigma_{\perp}^{R}-\eta_{\perp} \sigma_{\|}^{R}\right)\left[\cos \frac{x-x_{L}}{\lambda_{\Delta}^{L}}+\frac{\sigma_{\Delta}^{L} \eta_{\perp}}{\sigma_{\Delta}^{R} \chi_{\perp}} \sin \frac{x-x_{L}}{\lambda_{\Delta}^{L}}\right] \cos \theta \mathbf{p} \times(\mathbf{n} \times \mathbf{p}) \\
& +\frac{P j_{c}}{e^{3} \mathcal{N}} \frac{\mathrm{e}^{\left(x-x_{L}\right) / \lambda_{\perp}^{L}}}{D}\left(\eta_{\|} \sigma_{\perp}^{R}-\eta_{\perp} \sigma_{\|}^{R}\right)\left[\sin \frac{x-x_{L}}{\lambda_{\Delta}^{L}}-\frac{\sigma_{\Delta}^{L} \eta_{\perp}}{\sigma_{\Delta}^{R} \chi_{\perp}} \cos \frac{x-x_{L}}{\lambda_{\Delta}^{L}}\right] \cos \theta \mathbf{p} \times \mathbf{n},
\end{aligned}
$$

and in the antiferromagnet,

$$
\begin{aligned}
\mathbf{S}_{A}+\left.\mathbf{S}_{B}\right|_{x>x_{R}}= & \frac{P j_{\mathcal{c}}}{e^{3} \mathcal{N}} \frac{\mathrm{e}^{-\left(x-x_{R}\right) / \lambda_{\|}^{R}}}{D}\left(\sigma_{\perp}^{L}+\sigma_{\perp}^{R}+\frac{\eta_{\perp}^{2} \sigma_{\Delta}^{L 2}}{\chi_{\perp} \sigma_{\perp}^{R}}\right) \cos \theta \mathbf{n} \\
& +\frac{P j_{\mathcal{c}}}{e^{3} \mathcal{N}} \frac{\mathrm{e}^{-\left(x-x_{R}\right) / \lambda_{\perp}^{R}}}{D}\left(\eta_{\|} \sigma_{\perp}^{L}+\sigma_{\|}^{R}+\frac{\eta_{\perp} \eta_{\|} \sigma_{\Delta}^{L 2}}{\chi_{\perp} \sigma_{\perp}^{R}}\right) \mathbf{n} \times(\mathbf{p} \times \mathbf{n}) \\
& +\frac{P j_{c}}{e^{3} \mathcal{N}} \frac{\mathrm{e}^{-\left(x-x_{R}\right) / \lambda_{\perp}^{R}}}{D}\left(\eta_{\|} \sigma_{\perp}^{R}-\eta_{\perp} \sigma_{\|}^{R}\right) \cos \theta \mathbf{n} \times \mathbf{p} .
\end{aligned}
$$

We defined the denominator 


$$
\begin{aligned}
D= & \left(\eta_{\perp} \sigma_{\perp}^{L}+\sigma_{\perp}^{R}+\frac{\eta_{\perp}^{2} \sigma_{\Delta}^{L 2}}{\chi_{\perp} \sigma_{\perp}^{R}}\right)\left(\sigma_{\|}^{R}+\eta_{\|} \sigma_{\|}^{L}\right) \cos ^{2} \theta \\
& +\left(\eta_{\|} \sigma_{\perp}^{L}+\sigma_{\|}^{R}+\frac{\eta_{\perp} \eta_{\|} \sigma_{\Delta}^{L 2}}{\chi_{\perp} \sigma_{\perp}^{R}}\right)\left(\sigma_{\perp}^{R}+\eta_{\perp} \sigma_{\|}^{L}\right) \sin ^{2} \theta
\end{aligned}
$$

In the expressions above, $\theta$ is the angle between $\mathbf{n}$ and $\mathbf{p}$ $(\cos \theta=\mathbf{n} \cdot \mathbf{p}), \lambda_{\|}^{L, R}$ is the relaxation length for spins longitudinal to the magnetic order parameter, $\lambda_{\perp}^{L, R}$ is the relaxation length for spins transverse to it, and $\lambda_{\Delta}^{L}$ is the spin precession length in the ferromagnet. Here $L$ stands for the ferromagnet, while $R$ stands for the antiferromagnet. We also defined the interfacial spin conductivity $\sigma_{\|, \perp}^{L, R}=\mathcal{D}^{L, R} / e^{2} \mathcal{N} \lambda_{\|, \perp}^{L, R}$ (in units of $\Omega^{-1} \cdot \mathrm{m}^{-2}$ ), which quantifies the ability for the spin current to enter the (ferro- or antiferro-)magnetic metal. For instance, in the antiferromagnet, since spins longitudinal and transverse to the Néel order parameter relax with different rates $\left(\lambda_{\|}^{R}>\lambda_{\perp}^{R}\right)$, their spin conductivity is different $\left(\sigma_{\perp}^{R}>\sigma_{\|}^{R}\right)$. Finally, we also note $\eta_{\|, \perp}=1+r_{\text {int }} \sigma_{\|, \perp}^{R}$, a parameter that quantifies the influence of the interfacial resistance, and $\chi_{\perp}=1+\eta_{\perp} \sigma_{\perp}^{L} / \sigma_{\perp}^{R}$, which quantifies the conductivity mismatch between the ferromagnetic and antiferromagnetic metals.

Let us now comment on the non-equilibrium uniform spin density in the antiferromagnet, equation (36). It is interesting to notice that the uniform spin density injected from the ferromagnet into the antiferromagnet is mostly in-plane $(\sim \mathbf{n} \times(\mathbf{p} \times \mathbf{n}))$. Remarkably, the out-of-plane component $(\sim \mathbf{n} \times \mathbf{p})$ is proportional to $\eta_{\|} \sigma_{\perp}^{R}-\eta_{\perp} \sigma_{\|}^{R}$. In other words, the out-of-plane component of the spin density in the antiferromagnet comes from the anisotropy of the spin relaxation $\lambda_{\perp}^{R} \neq \lambda_{\|}^{R}$. In turn, this anisotropy induces a spin density in the ferromagnet that is transverse to the magnetization direction (second and third terms in equation (35)). This means that, in principle, due to the spin relaxation anisotropy in the antiferromagnet, a torque can be exerted from the antiferromagnet on the ferromagnet. Finally, we also note that the components of the spin density that are proportional to $\eta_{\|} \sigma_{\perp}^{R}-\eta_{\perp} \sigma_{\|}^{R}$ are also proportional to $\cos \theta$, i.e. they vanish when the ferromagnetic order parameter is orthogonal to the antiferromagnetic order parameter, a feature already noticed by Haney and MacDonald [19].

Let us now address the torque exerted by the ferromagnet on the antiferromagnet. By definition, the torque arising from the staggered spin density reads

$$
\begin{aligned}
\mathbf{T} & =2 \Delta \int_{x_{R}}^{+\infty} \mathrm{d} x\left(\mathbf{S}_{A}-\mathbf{S}_{B}\right) \times \mathbf{n} \\
& =\frac{\tau^{*} \hbar}{\tau_{\Delta}^{2}} \int_{x_{R}}^{+\infty} \mathrm{d} x \mathbf{n} \times\left[\left(\mathbf{S}_{A}+\mathbf{S}_{B}\right) \times \mathbf{n}\right],
\end{aligned}
$$

which yields

$$
\begin{aligned}
\mathbf{T}= & \lambda_{\perp}^{R} \frac{\tau^{*} \hbar}{\tau_{\Delta}^{2}} \frac{P j_{c}}{e^{3} \mathcal{N}} \frac{1}{D}\left(\eta_{\|} \sigma_{\perp}^{L}+\sigma_{\|}^{R}+\frac{\eta_{\perp} \eta_{\|} \sigma_{\Delta}^{L 2}}{\chi_{\perp} \sigma_{\perp}^{R}}\right) \mathbf{n} \times(\mathbf{p} \times \mathbf{n}) \\
& +\lambda_{\perp}^{R} \frac{\tau^{*} \hbar}{\tau_{\Delta}^{2}} \frac{P j_{c}}{e^{3} \mathcal{N}} \frac{1}{D}\left(\eta_{\|} \sigma_{\perp}^{R}-\eta_{\perp} \sigma_{\|}^{R}\right) \cos \theta \mathbf{n} \times \mathbf{p} .
\end{aligned}
$$

Again, the torque is dominated by an in-plane component and possesses a small out-of-plane component, the latter vanishing when the order parameters are orthogonal to each other. In the limit where the spin relaxation is isotropic in the antiferromagnet $\left(\lambda_{\perp}^{R} \approx \lambda_{\|}^{R}\right)$, the torque reduces to

$$
\mathbf{T}=\frac{\tau^{*} \hbar}{\tau_{\Delta}^{2}} \frac{1}{e^{3} \mathcal{N}} \frac{\lambda_{\|}^{R} P j_{c}}{\sigma_{\|}^{R}+\sigma_{\|}^{L}+r_{\mathrm{int}} \sigma_{\|}^{L} \sigma_{\|}^{R}} \mathbf{n} \times(\mathbf{p} \times \mathbf{n}) .
$$

The dynamics of the antiferromagnetic order parameter driven by an in-plane torque has been investigated by Gomonay [36, 37] and we refer the reader to these works for further details.

\subsection{Antiferromagnetic bilayer}

In the previous section, we showed that the spin torque arising from a ferromagnetic polarizer in a spin-valve configuration is efficient in manipulating the order parameter of an antiferromagnet, even in the diffusive limit. Yet, the fabrication of such a device remains challenging and a much simpler configuration is a magnetic bilayer that consists of an antiferromagnet deposited on top of a heavy metal $[49,50]$. In the heavy metal, spin-orbit coupling is large enough to enable spin Hall effect. This configuration has been recently investigated experimentally by Reichlová et al [18]. In the heavy metal, the uniform spin density fulfills the following transport equations $[47,51]$

$$
\begin{gathered}
\mathbf{J}_{i}^{s}=-\mathcal{D} \nabla_{i}\left(\mathbf{S}_{A}+\mathbf{S}_{B}\right)+\alpha_{\mathrm{H}} \mathcal{D} \mathbf{e}_{i} \times \nabla\left(n_{A}+n_{B}\right), \\
\partial_{t}\left(\mathbf{S}_{A}+\mathbf{S}_{B}\right)+\frac{1}{\tau_{\mathrm{sf}}}\left(\mathbf{S}_{A}+\mathbf{S}_{B}\right)=-\partial_{i} \mathbf{J}_{i}^{s},
\end{gathered}
$$

where the second term in equation (41) stands for the spin Hall effect induced by the charge gradient (or equivalently, an electric field). In the configuration depicted on figure 2(c), we consider that the electric field is applied along $x$, i.e. $\nabla\left(n_{A}+n_{B}\right)=-\mathcal{N} e E \mathbf{x}$, while the normal to the interface is along $z$. The heavy metal has a thickness $d$, while the antiferromagnet is much thicker than its spin relaxation length. We also consider that there is an interfacial resistivity $r_{\text {int }}$ between the two layers, at $z=0$. As already mentioned, one can introduce interfacial spin-flip by adding a fictitious interfacial layer [48], but this makes our analytical results significantly more complicated. We assume that there is no spin current flowing through the outer boundary of the heavy metal $\left(\left.\mathcal{J}_{z}^{s}\right|_{z=-d}=0\right)$. Therefore, one simply needs to connect the spin current and densities at the interface between the antiferromagnet and the heavy metal, and we obtain 


$$
\begin{aligned}
\mathbf{S}_{A}+\left.\mathbf{S}_{B}\right|_{x>0}= & \frac{\alpha_{\mathrm{H} j}}{e^{3} \mathcal{N}} \frac{\mathrm{e}^{-x / \lambda_{\|}^{R}}}{\sigma_{\|}^{R}+\eta_{\|} \sigma_{\mathrm{sf}}^{L} \tanh \frac{d}{\lambda_{\mathrm{sf}}^{L}}} n_{y}\left(1-\cosh ^{-1} \frac{d}{\lambda_{\mathrm{sf}}^{L}}\right) \mathbf{n} \\
& +\frac{\alpha_{\mathrm{H} j}}{e^{3} \mathcal{N}} \frac{\mathrm{e}^{-x / \lambda_{\perp}^{R}}}{\sigma_{\perp}^{R}+\eta_{\perp} \sigma_{\mathrm{sf}}^{L} \tanh \frac{d}{\lambda_{\mathrm{sf}}^{L}}}\left(1-\cosh ^{-1} \frac{d}{\lambda_{\mathrm{sf}}^{L}}\right) \mathbf{n} \\
& \times(\mathbf{p} \times \mathbf{n}) .
\end{aligned}
$$

One can remark that the uniform spin density in the antiferromagnet does not possess an out-of-plane component $(\sim \mathbf{n} \times \mathbf{p})$, in contrast to the spin-valve case studied above. The resulting torque reads

$\mathbf{T}=\frac{\tau^{*} \hbar}{\tau_{\Delta}^{2}} \frac{1}{e^{3} \mathcal{N}} \frac{\lambda_{\perp}^{R} \alpha_{\mathrm{H}} j_{c}}{\sigma_{\perp}^{R}+\eta_{\perp} \sigma_{\mathrm{sf}}^{L} \tanh \frac{d}{\lambda_{\mathrm{sf}}^{L}}}\left(1-\cosh ^{-1} \frac{d}{\lambda_{\mathrm{sf}}^{L}}\right) \mathbf{n} \times(\mathbf{p} \times \mathbf{n})$.

The structure of this expression is very similar to equation (40), which comes as no surprise since the only distinction between the spin-valve and the bilayer structures comes from the nature of the spin polarization (either from a ferromagnetic polarizer or from spin Hall effect).

\subsection{Self-torque in single antiferromagnets}

Let us now turn our attention towards the fourth case represented on figure 2(d), the homogeneous antiferromagnet. We now solve equation (31) by assuming that spin Hall effect is present in the antiferromagnet. Spin Hall effect does not modify the spin diffusion equation, but rather the spin current definition. Since an antiferromagnet behaves essentially like a normal metal, it is sufficient to expend the definition of the spin current, equation (29), by accounting for spin Hall effect

$$
\mathbf{J}_{i}^{s}=-\mathcal{D} \nabla_{i}\left(\mathbf{S}_{A}+\mathbf{S}_{B}\right)+\alpha_{\mathrm{H}} \mathcal{D} \mathbf{e}_{i} \times \nabla\left(n_{A}+n_{B}\right),
$$

where we neglected the anisotropy of the diffusion coefficients for simplicity. To ensure that an effective torque applies on the system, one has to make the boundary conditions asymmetric, otherwise no effective torque survives after averaging out over the sample volume. For instance, we assume a thickness $d$, such that at $z=0$ the spin density vanishes, while at $z=d$ the spin current vanishes. Therefore, the uniform spin density reads

$$
\mathbf{S}_{A}+\mathbf{S}_{B}=\frac{1}{e^{3} \mathcal{N}} \frac{\alpha_{\mathrm{H} j}}{\sigma_{\perp}} \frac{\sinh \frac{z}{\lambda_{\perp}}}{\cosh \frac{z}{\lambda_{\perp}}} \mathbf{n} \times(\mathbf{y} \times \mathbf{n}),
$$

and its associated torque is

$$
\mathbf{T}=\frac{\tau^{*} \hbar}{\tau_{\Delta}^{2}} \frac{1}{e^{3} \mathcal{N}} \frac{\lambda_{\perp} \alpha_{\mathrm{H} j}}{\sigma_{\perp}}\left(1-\cosh ^{-1} \frac{d}{\lambda_{\perp}}\right) \mathbf{n} \times(\mathbf{y} \times \mathbf{n}) .
$$

Of course the exact expression depends on the exact boundary conditions at $z=0$ and $z=d$. Experimentally, the symmetry can be broken by imposing strong spin-flip at one interface (by dusting the surface with heavy metal impurities for instance) and weak spin-flip at the other interface (using either a light metal like $\mathrm{Cu}$ or a tunnel barrier like $\mathrm{MgO}$ ). Finally, noticing that $\lambda_{\perp} / e^{2} \mathcal{N} \sigma_{\perp}=\tau_{\perp}$ is the transverse spin relaxation time, we remark that the three torques derived in this work have the same general expression

Polarization $\cdot \frac{\text { Life time }}{\text { Precession time }} \cdot \frac{\text { Transverse relaxation time }}{\text { Precession time }}$,

where 'Polarization' refers to the spin polarization of the incoming spin current (either from direct spin injection $\sim P$ or from spin Hall effect, $\sim \alpha_{\mathrm{H}}$ ), 'Life time' refers to the time $\tau^{*}$ an electron spends on one sublattice, 'Precession time' refers to the time $\tau_{\Delta}$ an electron spin takes to undertake a full revolution about the local magnetic moment, and finally 'transverse relaxation time' is the time $\tau_{\perp}$ after which the spin component transverse to the local order parameter is lost.

\section{Conclusion}

We have developed a drift-diffusion theory of spin transport in collinear bipartite metallic antiferromagnets. Using equations (28)-(32), we derived an expression of the spin transfer torque exerted on the antiferromagnetic free layer in three experimentally-relevant configurations: (i) a spin-valve composed of a ferromagnet and an antiferromagnet, (ii) a metallic bilayer consisting in an antiferromagnet adjacent to a heavy metal possessing spin Hall effect, and in (iii) a single antiferromagnet possessing spin Hall effect. We show that in all three cases, the uniform torque arising from the staggered spin density that lies in the $(\mathbf{n}, \mathbf{p})$ plane, $\sim \mathbf{n} \times(\mathbf{p} \times \mathbf{n})$.

This work shows that spin transfer torque in spin devices involving antiferromagnets survives in the diffusive regime, which is quite promising for potential applications. Several issues remain to be solved though $[12,13]$. The electrical detection of the Néel order dynamics is probably the most significant challenge, although recent breakthroughs exploiting anisotropic magnetoresistance are encouraging [16]. A second problem is the quality of the interface between antiferromagnets and normal metals and in particular the nature of the antiferromagnetic spin texture at this interface [3]. Finally, the present work is limited to the archetypal collinear G-type antiferromagnet whereas the most common metallic antiferromagnets, such as IrMn, possess a non-collinear antiferromagnetic texture. Extending the present model to such non-collinear antiferromagnets and understanding their current-induced dynamics remain to be explored carefully.

\section{Acknowledgments}

This work was supported by the King Abdullah University of Science and Technology (KAUST) through the Office of Sponsored Research (OSR) (Grant Number OSR2015-CRG4-2626). The author acknowledges inspiring discussions with T Jungwirth, J Sinova, J Zelezny, H Gomonay and H Saidaoui. 


\section{References}

[1] Néel L 1932 Ann. Phys. 175

[2] Néel L 1970 Nobel Lect. Phys. 1963-70

[3] Nogues J and Schuller I K 1999 J. Magn. Magn. Mater. 192 203-32

[4] Dieny B, Speriosu V S, Parkin S S P, Gurney B A, Wilhoit D R and Mauri D 1991 Phys. Rev. B 431297

[5] Giamarchi T, Rüegg C and Tchernyshyov O 2008 Nat. Phys. 4198

[6] Mourigal M, Enderle M, Klöpperpieper A, Caux J-S, Stunault A and Rnnow H M 2013 Nat. Phys. 9435

[7] Merchant P, Normand B, Krämer K W, Boehm M, McMorrow D F and Rüegg Ch 2014 Nat. Phys. 10373

[8] Núňez A S, Duine R A, Haney P and MacDonald A H 2006 Phys. Rev. B 73214426

[9] Park B G et al 2011 Nat. Mater. 10347

[10] Wang Y Y, Song C, Cui B, Wang G Y, Zeng F and Pan F 2012 Phys. Rev. Lett. 109137201

[11] Manchon A, Koo H C, Nitta J, Frolov S M and Duine R A 2015 Nat. Mater. 14271

[12] Jungwirth T, Martí X, Wadley P and Wunderlich J 2016 Nat. Nanotechnol. 11231

[13] Baltz V, Manchon A, Tsoi M, Moriyama T, Ono T and Tserkovnyak Y 2016 arXiv:1606.04284

[14] Urazhdin S and Anthony N 2007 Phys. Rev. Lett. 99046602

[15] Wei Z, Sharma A, Nunez A S, Haney P M, Duine R A, Bass J, MacDonald A H and Tsoi M 2007 Phys. Rev. Lett. 98116603

[16] Wadley P et al 2016 Science 351 587-90

[17] Železný J, Gao H, Výborný K, Zemen J, Mašek J, Manchon A, Wunderlich J, Sinova J and Jungwirth T 2014 Phys. Rev. Lett. 113157201

Železný J et al 2017 Phys. Rev. B 95014403

[18] Reichlová H et al 2015 Phys. Rev. B 92165424

[19] Haney P M and MacDonald A H 2008 Phys. Rev. Lett. 100196801

[20] Duine R A, Haney P M, Núñez A S and MacDonald A H 2007 Phys. Rev. B 75014433

[21] Cheng R and Niu Q 2012 Phys. Rev. B 86245118

[22] Prakhya K, Popescu A and Haney P M 2014 Phys. Rev. B 89054421

[23] Saidaoui H B, Waintal X and Manchon A 2014 Phys. Rev. B 89174430

[24] Merodio P, Kalitsov A, Béa H, Baltz V and Chshiev M 2014 Appl. Phys. Lett. 105122403

[25] Saidaoui H B, Waintal X and Manchon A 2016 arXiv: 1607.01523

[26] Saidaoui H B and Manchon A 2016 arXiv:1606.04261

[27] Haney P M, Waldron D, Duine R A, Núñez A S, Guo H and MacDonald A H 2007 Phys. Rev. B 75174428

[28] Xu Y, Wang S and Xia K 2008 Phys. Rev. Lett. 100226602
[29] Brataas A, Nazarov Y V and Bauer G E W 2000 Phys. Rev. Lett. 842481

Brataas A, Nazarov Y V and Bauer G E W 2001 Eur. Phys. J. B 22 99-110

[30] Brataas A, Bauer G E W and Kelly P J 2006 Phys. Rep. 427 157-255

[31] Cheng R, Xiao J, Niu Q and Brataas A 2014 Phys. Rev. Lett. 113057601

[32] Takei S, Halperin B I, Yacoby A and Tserkovnyak Y 2014 Phys. Rev. B 90094408

[33] Valet T and Fert A 1993 Phys. Rev. B 487099

[34] Zhang S, Levy P M and Fert A 2002 Phys. Rev. Lett. 88236601

[35] Petitjean C, Luc D and Waintal X 2012 Phys. Rev. Lett. 109117204

[36] Gomonay E V and Loktev V M 2010 Low Temp. Phys. 34198

Gomonay E V and Loktev V M 2008 Phys. Rev. B 81144427

[37] Gomonay E V and Loktev V M 2014 Low Temp. Phys. 4017

[38] Baryakhtar I V and Ivanov B A 1979 Fiz. Nizk. Temp. 5759 Baryakhtar I V and Ivanov B A 1979 Sov. J. Low Temp. Phys. 5361

[39] Kimel A V, Kirilyuk A, Tsvetkov A, Pisarev R V and Rasing T 2004 Nature 429850

[40] Wienholdt S, Hinzke D and Nowak U 2012 Phys. Rev. Lett. 108247207

[41] Kovalev A A, Výborný K and Sinova J 2008 Phys. Rev. B $78041305 R$

[42] Mishchenko E G, Shytov A V and Halperin B I 2004 Phys. Rev. Lett. 93226602

[43] Wang X and Manchon A 2012 Phys. Rev. Lett. 108117201 Wang W, Pauyac C O and Manchon A 2014 Phys. Rev. B 89054405

[44] Shen K, Raimondi R and Vignale G 2014 Phys. Rev. B 90245302

[45] Ndiaye P B, Akosa C A, Fischer M H, Vaezi A, Kim E-A and Manchon A 2015 arXiv:1509.06929

[46] Pauyac C O, Nikolaev S, Chshiev M and Manchon A in preparation

[47] Haney P M, Lee H-W, Lee K-J, Manchon A and Stiles M D 2013 Phys. Rev. B 87174411

[48] Liu Y, Yuan Z, Wesselink R J H, Starikov A A and Kelly P J 2014 Phys. Rev. Lett. 113207202

[49] Miron I M et al 2010 Nat. Mater. 9230

Liu L, Moriyama T, Ralph D C and Buhrman R A 2011 Phys. Rev. Lett. 106036601

Miron I M et al 2011 Nature 476 189-93

Liu L, Pai C-F, Li Y, Tseng H W, Ralph D C and Buhrman R A 2012 Science 336555

[50] Uchida K et al 2010 Nat. Mater. 9894

[51] Shchelushkin R V and Brataas A 2005 Phys. Rev. B 71045123 